\title{
LINK CLOSED SETS OF PRIME IDEALS AND STABILITY ON BIMODULES
}

\author{
Karl A. Kosler \\ Received: 29 June 2014; Revised: 12 September 2014 \\ Communicated by Abdullah Harmancı
}

\begin{abstract}
Over a Noetherian ring $R$, each of a weakly symmetric pair of torsion radicals is shown to be stable on Noetherian $R$ - $R$ bimodules if and only if the set of prime ideals that are closed with respect to each torsion radical is closed under links. Such a pair for $R$, termed a weakly symmetric stable pair, is extended to a weakly symmetric stable pair for any Noetherian extension ring of $R$. In case classical Krull dimension is a link invariant, we give a positive answer to the incomparability question for linked prime ideals in certain extension rings of $R$.
\end{abstract}

Mathematics Subject Classification (2010): 16D20, 16D25, 16S99

Keywords: Noetherian ring, extension ring, prime ideal, link, torsion radical, classical Krull dimension

\section{Introduction}

For a ring $R$, a torsion radical is a hereditary (left exact) functor defined on the category $R$-Mod or Mod- $R$ of left or right $R$-modules as given in [4] or [10]. A pair of torsion radicals is an ordered pair $(\sigma, \tau)$ where $\sigma$ is a torsion radical defined on $R$-Mod and $\tau$ is a torsion radical defined on $\operatorname{Mod}-R$. A pair $(\sigma, \tau)$ is called weakly symmetric if $\sigma(R / P)=\tau(R / P)$ for all prime ideals $P$. In this case, $\operatorname{Sp}(\sigma, \tau)$ denotes the set of all $\sigma$-dense $(\tau$-dense) prime ideals of $R$. A pair $(\sigma, \tau)$ is called stable provided, for any $\operatorname{ring} \Lambda, \sigma$ and $\tau$ are stable on Noetherian $R$ - $\Lambda$ bimodules and Noetherian $\Lambda-R$ bimodules respectively. The aim of first part of this paper is to show that when all rings involved are two-sided Noetherian, a weakly symmetric stable pair can be characterized internally as one for which the set of dense prime ideals $\operatorname{Sp}(\sigma, \tau)$ is closed under links.

The rest of the paper examines the problem of lifting a stable pair to an extension ring $S \supseteq R$ with the idea of generating a link-closed set of prime ideals in $S$ starting from a link-closed set of prime ideals in $R$. In particular, it is shown that if $S \supseteq R$ are both Noetherian and $(\sigma, \tau)$ is a weakly symmetric stable pair for $R$, then the canonical extension $\left(\sigma^{*}, \tau^{*}\right)$ is a weakly symmetric stable pair for $S$. Thus, a link 
closed set of prime ideals of $R$ of the form $\operatorname{Sp}(\sigma, \tau)$ gives rise to a link closed set $\operatorname{Sp}\left(\sigma^{*}, \tau^{*}\right)$ of prime ideals of $S$. We use this result to show that if $R$ is a Noetherian ring where classical Krull dimension is a link invariant, then for linked prime ideals $P, Q$ of $S, R /(P \cap R)$ and $R /(Q \cap R)$ have equal classical Krull dimension. As a corollary, linked prime ideals in a Noetherian extension ring $S$ are incomparable provided $S$ is either a finite normalizing extension of $R$ or a strongly $G$-graded ring with base ring $R$ for some finite group $G$.

\section{Notation and definitions}

All rings considered have an identity and modules are unitary. Properties and adjectives, when not accompanied by one-handed modifiers are meant to hold on both sides. However, all modules will be left $R$-modules unless otherwise specified. The notation $N \leq{ }_{R} M$, or $N \leq M$ when $R$ is understood, will be used to indicate that $N$ is a (left) $R$-submodule of $M$. Similarly for $N \leq M_{R}$. If $N \leq M$ is essential in $M$, then we will write $N \leq_{e} M$. In case $M$ is an $S-R$ bimodule, we will write ${ }_{S} M_{R}$ and use $N \leq{ }_{S} M_{R}$ to indicate that $N$ is an $S$ - $R$ subbimodule of $M$.

For a nonempty subset $X$ of an $R$-module $M, l_{R}(X)=\{r \in R \mid r x=0$ for all $x \in X\}$. In case $X$ is a nonempty subset of a left $R$-module, then $r_{R}(X)=\{r \in R \mid$ $x r=0$ for all $x \in X\}$. If $X$ is a nonempty subset of $R$ and $M$ is a left $R$-module, then $\operatorname{ann}_{M}(X)=\{m \in M \mid x m=0$ for all $x \in X\}$. A module $M$ is said to be finitely annihilated if there exist finitely many elements $m_{1}, \ldots, m_{k} \in M$ such that $l_{R}(M)=l_{R}\left(m_{1}, \ldots, m_{k}\right)$. Note that if $M$ is a finitely annihilated module, then there is a one-to-one map $R / l_{R}(M) \rightarrow M^{(n)}$ for some $n$.

The collection of all prime ideals of $R$ is denoted by $\operatorname{Spec}(R)$. If $U$ is a uniform $R$-module, and $R$ is left Noetherian, then it follows from [5, Lemma 4.22] that there is a unique prime ideal $P$ such that all nonzero $X \leq \operatorname{ann}_{U}(P)$ have $l_{R}(X)=P$. The ideal $P$ is called the associated prime ideal (or assassinator) of $U$ and is denoted by $\operatorname{ass}(U)$. For an arbitrary module $M$, the set of associated prime ideals of $M$, denoted by $\operatorname{Ass}(M)$, is the set of all prime ideals ass $(U)$ where $U$ is a uniform submodule of $M$. The set of maximal members of $\operatorname{Ass}(M)$ will be denoted by $\max \operatorname{Ass}(M)$.

If $P \in \operatorname{Spec}(R)$ and $\sigma$ is a torsion radical defined on $R$-Mod or Mod- $R$, then $P$ is called $\sigma$-dense provided $R / P$ is $\sigma$-torsion while $P$ is called $\sigma$-closed provided $R / P$ is $\sigma$-torsion-free. The set of all $\sigma$-dense prime ideals is denoted by $\operatorname{Spec}^{\sigma}(R)$, and the set of all $\sigma$-closed prime ideals is denoted by $\operatorname{Spec}_{\sigma}(R)$. It is well known that a prime ideal $P$ of a left Noetherian ring $R$ is either $\sigma$-dense or $\sigma$-closed in case $\sigma$ is defined on $R$-Mod. 


\section{Stability and linked primes}

Throughout this section, $R$ will denote a two-sided Noetherian ring. If $\mathcal{C}$ is a class of $R-\Lambda$ bimodules for some ring $\Lambda$ and $\sigma$ is a torsion radical defined on $R$-mod, then we say that $\sigma$ is stable on $\mathcal{C}$ provided that for all $M \in \mathcal{C}, \sigma(M)$ is an essentially closed left $R$-submodule of $M$. Equivalently, $\sigma$ is stable on $\mathcal{C}$ provided that for all $M \in \mathcal{C}, \sigma(M) \leq_{e R} M$ implies that $\sigma(M)=M$. Again, the right-hand version is defined in the obvious way.

The aim of this section is to show that for a pair $(\sigma, \tau)$ with $\operatorname{Spec}^{\sigma}(R)=$ $\operatorname{Spec}^{\tau}(R)$, stability on Noetherian bimodules is equivalent to the closure of this class of prime ideals under links. For $P, Q \in \operatorname{Spec}(R)$, there is a link from $Q$ to $P$, denoted $Q \sim \rightarrow P$ provided there exists an ideal $A$ of $R$ with $Q P \subseteq A \subset Q \cap P$ such that $(Q \cap P) / A$ is torsion-free as a left $R / Q$-module and as a right $R / P$-module $($ see $[5])$.

Proposition 3.1. Let $(\sigma, \tau)$ be a pair of torsion radicals.

(1) If $\sigma$ is stable on ideal factors of $R$ and if $Q \in \operatorname{Spec}^{\sigma}(R)$ and $Q \sim \rightarrow P$, then $P \in \operatorname{Spec}^{\sigma}(R)$.

(2) If $\tau$ is stable on ideal factors of $R$ and if $P \in \operatorname{Spec}^{\tau}(R)$ and $Q \sim \rightarrow P$, then $Q \in \operatorname{Spec}^{\tau}(R)$.

Proof. Let $A$ be an ideal with $Q P \subseteq A \subset Q \cap P$ such that $(Q \cap P) / A$ is torsionfree on both sides. Let $I / A \neq 0$ be a left ideal of $R / A$. If $P I \nsubseteq A$, then $0 \neq$ $(P I+A) / A \subseteq(P / A) \cap(I / A)$. If $P I \subseteq A$, then $I \subseteq r_{R}(P / A) \subseteq r_{R}((Q \cap P) / A)=P$ where the last equality follows since $(Q \cap P) / A$ is torsion-free over $R / P$. In either case, $(P / A) \cap(I / A) \neq 0$. Thus, $P / A \leq_{e R} R / A$. Note that $Q / A \leq_{e} R / A_{R}$ by the symmetric argument.

For (1), since $Q P \subseteq A$ and $Q$ is $\sigma$-dense, $P / A$ is $\sigma$-torsion. Thus, $\sigma(R / A) \leq_{e R} R / A$, hence $R / A$ is $\sigma$-torsion. Therefore, $R / P$ is $\sigma$ torsion i.e. $P$ is $\sigma$-dense.

For (2), $Q P \subseteq A$ and $P \tau$-dense implies that $Q / A$ is $\tau$-torsion. The conclusion follows as in (1).

A nonempty subset $\Omega$ of $\operatorname{Spec}(R)$ is right link closed provided $P \in \Omega$ whenever $Q \sim \rightarrow P$ for some $Q \in \Omega$. Likewise, a nonempty subset $\Omega$ of $\operatorname{Spec}(R)$ is left link closed provided $Q \in \Omega$ whenever $Q \sim \rightarrow P$ for some $P \in \Omega$. A nonempty subset $\Omega$ of $\operatorname{Spec}(R)$ is link closed if it is both right and left link-closed. Thus, from Proposition 3.1 , if $(\sigma, \tau)$ is a pair of torsion radicals with $\operatorname{Spec}^{\sigma}(R)=\operatorname{Spec}^{\tau}(R)$ and both $\sigma$ and $\tau$ are stable on ideal factors of a Noetherian $\operatorname{ring} R$, then $\operatorname{Spec}^{\sigma}(R)$ is link closed. 
Below, we show that the converse is true and, in this case, stability extends to a much larger class of bimodules.

Lemma 3.2. Let ${ }_{R} B_{\Lambda} \neq 0$ be a right Noetherian bimodule where $\Lambda$ is any ring.

(1) Every left $R$-submodule of $B$ is finitely annihilated.

(2) If $\sigma$ is a torsion radical and $\sigma(B)=B$, then every annihilator ideal of $B$ is $\sigma$-dense.

(3) If $\sigma$ is a torsion radical and $\sigma(B)=0$, then every annihilator ideal of $B$ is $\sigma$-closed.

(4) Let $P \in \operatorname{Ass}\left({ }_{R} B\right)$. Then $\operatorname{ann}_{B}(P)$ is a torsion-free left $R / P$-module iff $P \in \operatorname{maxAss}\left({ }_{R} B\right)$.

(5) If $P \in \operatorname{maxAss}\left({ }_{R} B\right)$ and $Q \in \operatorname{Ass}\left({ }_{R} B / \operatorname{ann}_{B}(P)\right)$, then $Q \in \operatorname{Ass}\left({ }_{R} B\right)$ or $Q \sim \rightarrow P$.

Proof. (1) Pick a $0 \neq F \leq{ }_{R} B$. As a right $\Lambda$-module, $F \Lambda$ is Noetherian and so $F \Lambda=x_{1} \Lambda+\ldots+x_{m} \Lambda$ for some $x_{1}, \ldots, x_{m} \in F$. It follows that $l_{R}(F) \subseteq$ $l_{R}\left(x_{1}, \ldots, x_{m}\right) \subseteq l_{R}(F)$. Therefore, $l_{R}(F)=l_{R}\left(x_{1}, \ldots, x_{m}\right)$.

(2), (3) Let $A=l_{R}(F)$ where $0 \neq F \leq{ }_{R} B$. By (1), there is a one-to-one map $R / A \rightarrow F^{(k)}$ for some $k$. As $B$ is $\sigma$-torsion or $\sigma$-torsionfree, then respectively, so is $F^{(k)}$. The conclusion follows.

(4) If $\operatorname{ann}_{B}(P)$ is a torsion-free left $R / P$-module and $P \subset Q \in \operatorname{Ass}\left({ }_{R} B\right)$, then $0 \neq \operatorname{ann}_{B}(Q) \subseteq \operatorname{ann}_{B}(P)$. Since $(Q / P)\left(\operatorname{ann}_{B}(Q)\right)=0, \operatorname{ann}_{B}(Q)$ is a torsion $R / P$-submodule of $\operatorname{ann}_{B}(P)$, a contradiction.

Suppose that $P \in \operatorname{maxAss}\left({ }_{R} B\right)$. Let $Z \neq 0$ be the torsion $R / P$-submodule of $\operatorname{ann}_{B}(P)$. Let $Q \in \operatorname{Ass}\left({ }_{R} Z\right)$. By (1), $Z$ is finitely annihilated. It follows that $P \subset l_{R}(Z) \subseteq Q$ contradicting our choice of $P$.

(5) The last result is [7, Proposition 3.1].

A pair of torsion radicals $(\sigma, \tau)$ with $\operatorname{Spec}^{\sigma}(R)=\operatorname{Spec}^{\tau}(R)$ will be called weakly symmetric. In this case, we will denote the set of all $\sigma$-dense ( $\tau$-dense) prime ideals by $\operatorname{Sp}(\sigma, \tau)$.

Proposition 3.3. Let $(\sigma, \tau)$ be a weakly symmetric pair. If $\operatorname{Sp}(\sigma, \tau)$ is link closed, then $\sigma$ is stable on all Noetherian $R-\Lambda$ bimodules and $\tau$ is stable on all Noetherian $\Lambda-R$ bimodules.

Proof. If suffices to prove the first statement since the second follows by the symmetric argument. Let ${ }_{R} B_{\Lambda}$ be a Noetherian bimodule with $\sigma(B) \leq_{e} B$. Then $\operatorname{Ass}\left({ }_{R} B\right)=\operatorname{Ass}(\sigma(B))$ and so by Lemma 3.2(2), every member of $\operatorname{Ass}\left({ }_{R} B\right)$ is $\sigma$ dense. 
If $\sigma(B) \neq B$, then by Noetherian induction, we can assume that the result holds true for all proper bimodule factors of $B$. Pick $P \in \operatorname{maxAss}\left({ }_{R} B\right)$. Then $P$ is $\sigma$-dense and so $\operatorname{ann}_{B}(P) \subseteq \sigma(B) \subset B$.

If $\sigma(B)=\operatorname{ann}_{B}(P)$, then $B / \operatorname{ann}_{B}(P)$ is $\sigma$-torsionfree. Pick $Q \in \operatorname{Ass}\left(B / \operatorname{ann}_{B}(P)\right)$. Then $Q$ is $\sigma$-closed by Lemma 3.2(3) . Also, by Lemma 3.2(5), $Q \in \operatorname{Ass}\left({ }_{R} B\right)$ or $Q \sim \rightarrow P$. Clearly, the former fails and, by hypothesis, so does the latter. It follows that $\sigma(B) \supset \operatorname{ann}_{B}(P)$.

If $\sigma(B) / \operatorname{ann}_{B}(P)$ is an essential submodule of $B / \operatorname{ann}_{B}(P)$, then the conclusion follows from the inductive hypothesis. Suppose then that $\sigma(B) \cap X=\operatorname{ann}_{B}(P)$ for some submodule $X \supset \operatorname{ann}_{B}(P)$. Now, $\sigma(X)=\sigma(B) \cap X=\operatorname{ann}_{B}(P)$ and so $X / \operatorname{ann}_{B}(P)$ is $\sigma$-torsionfree. Choose $Q \in \operatorname{Ass}\left(X / \operatorname{ann}_{B}(P)\right) \subset \operatorname{Ass}\left(B / \operatorname{ann}_{B}(P)\right)$. By Lemma 3.2(3), $Q$ is $\sigma$-closed. However, by Lemma 3.2(5) again, $Q \in \operatorname{Ass}(R B)$ or $Q \sim \rightarrow P$. As before, both alternatives fail. Therefore, $\sigma(B)=B$.

A pair of torsion radicals $(\sigma, \tau)$ such that $\sigma$ is stable on all Noetherian $R-\Lambda$ bimodules and $\tau$ is stable on all Noetherian $\Lambda$ - $R$ bimodules for any ring $\Lambda$ will be called a stable pair.

Corollary 3.4. Let $(\sigma, \tau)$ be a weakly symmetric pair. The following are equivalent.

(1) $(\sigma, \tau)$ is a stable pair.

(2) $\sigma$ and $\tau$ are stable on ideal factors of $R$.

(3) $\sigma$ and $\tau$ are stable on all Noetherian $R$ - $R$ bimodules.

(4) $\operatorname{Sp}(\sigma, \tau)$ is link closed.

Proof. (1) $\Rightarrow(3)$ and (3) $\Rightarrow(2)$ are trivial. (2) $\Rightarrow$ (4) follows from Proposition 3.1 while $(4) \Rightarrow(1)$ is Proposition 3.3 .

\section{Ring extensions}

Let $S \supseteq R$ be a ring extension and let $\sigma$ be a torsion radical defined on $R$-mod. From [2, Ch.II(4)], the set

$$
\mathcal{D}=\left\{J \leq{ }_{S} S \mid \sigma\left({ }_{R} S / J\right)=S / J\right\}
$$

is an idempotent filter. This defines a torsion radical $\sigma^{*}$ on $S$-mod given by

$$
\sigma^{*}(M)=\{x \in M \mid J x=0 \text { for some } J \in \mathcal{D}\}
$$

It is easy to see that for any left $S$-module $M, \sigma^{*}(M)$ is the unique largest $S$ submodule of $M$ that is $\sigma$ torsion as a left $R$-module. Furthermore, if $J \in \mathcal{D}$, then $R \cap J$ is $\sigma$-dense. However, a left ideal of $S$ that contracts to a $\sigma$-dense left ideal of 
$R$ is not necessarily a member of $\mathcal{D}$. Note though that the situation is different for two sided ideals; see Lemma 4.2.

Below, we show that stability of $\sigma$ on bimodules passes to $\sigma^{*}$.

Theorem 4.1. Let $S \supseteq R$ be a Noetherian extension of a Noetherian ring $R$ and let $\sigma$ be a torsion radical. If $\sigma$ is stable on Noetherian $R-\Lambda$ bimodules, then $\sigma^{*}$ is stable on Noetherian $S-\Lambda$ bimodules.

Proof. Let $B$ be a Noetherian $S-\Lambda$ bimodule with $\sigma^{*}(B) \leq_{e S} B$. We claim that $\sigma(B) \leq_{e} B$. Clearly, it suffices to show that every member of $\operatorname{Ass}\left({ }_{R} B\right)$ is $\sigma$-dense.

Pick $P \in \operatorname{Ass}\left({ }_{R} B\right)$. Then there is a uniform submodule $0 \neq U \leq{ }_{R} B$ such that all $0 \neq X \leq \operatorname{ann}_{U}(P)$ satisfy $P=l_{R}(X)$. Let $V=\operatorname{ann}_{U}(P)$. By Lemma $3.2(1), V$ is finitely annihilated as a left $R$-module, hence there is a one-to-one map $R / P \rightarrow V^{(m)}$ for some $m$. By [5, Proposition 6.11], $V$ must be torsion-free as an $R / P$-module.

Consider the set $\Omega=\left\{l_{S}(S X) \mid 0 \neq X \leq{ }_{R} V\right\}$. Pick $D=l_{S}(S X) \in \Omega$ maximal. We claim that $D$ is a prime ideal of $S$. To this end, let $I, J$ be ideals of $S$ such that $I J \subseteq D$ and suppose that $J \nsubseteq D$. Then $J X=J S X=S(J X) \neq 0$ and $D \subseteq l_{S}(J X)$. By the maximality of $D, D=l_{S}(J X)$. Since $I J \subseteq D=l_{S}(S X)$, $I J S X=I J X=0$ and so $I \subseteq D$. Therefore, $D$ is a prime ideal of $S$.

By Lemma 3.2(1), $S X$ is finitely annihilated as a left $S$-module. Thus, there is a one-to-one $S$-module map $S / D \rightarrow(S X)^{(n)}$ for some $n$. Restricting this map to a nonzero uniform left ideal of $S / D$ yields an isomorphism onto a nonzero uniform $F \leq{ }_{S} S X$ which is clearly torsion-free over $S / D$. Since $\sigma^{*}(B) \leq_{e S} B, \sigma^{*}(F)=$ $\sigma^{*}(B) \cap F \neq 0$. Then $F$ torsion-free over $S / D$ forces $D=l_{S}\left(\sigma^{*}(F)\right)$. By Lemma $3.2(2), D$ is $\sigma^{*}$-dense. Then $D=l_{S}(S X)$ forces $S X$ to be $\sigma^{*}$-torsion. It follows that $\sigma(X)=X$. Now, from above, $P=l_{R}(X)$. Using Lemma 3.2(2) again, $P$ is $\sigma$-dense. Therefore, $\sigma(B) \leq_{e} B$, and so $\sigma(B)=B$. Since $\sigma^{*}(B)$ is the largest $S$-submodule of $B$ that is $\sigma$-torsion, $\sigma^{*}(B)=B$.

Next, we show that a weakly symmetric stable pair $(\sigma, \tau)$ for $R$ extends to a stable pair for $S$.

Lemma 4.2. Let $\sigma$ be a torsion radical defined on $R$-mod and let $S \supseteq R$ be a ring extension. Let $I \subseteq S$ be an ideal. Then $I$ is $\sigma^{*}$-dense if and only if $I \cap R$ is $\sigma$-dense.

Proof. From the definition of $\sigma^{*}$, the forward implication is obvious. For the reverse implication, let $I \subseteq S$ be an ideal such that $I \cap R$ is $\sigma$-dense. Fix $s \in S$ and define an epimorphism $R /(I \cap R) \rightarrow(R s+I) / I$ via right multiplication by 
s. (Note that this map is well defined since $I$ is an ideal of $S$.) It follows that for all $s \in S,(R s+I) / I$ is $\sigma$-torsion whence $S / I$ is $\sigma$-torsion as a left $R$-module. Therefore, $\sigma^{*}(S / I)=S / I$.

Corollary 4.3. Let $S \supseteq R$ be a Noetherian extension of a Noetherian ring $R$. Let $(\sigma, \tau)$ be a weakly symmetric stable pair for $R$. Then $\left(\sigma^{*}, \tau^{*}\right)$ is a weakly symmetric stable pair for $S$.

Proof. By Theorem 4.1, $\sigma^{*}$ is stable on Noetherian $S$ - $\Lambda$ bimodules for any ring $\Lambda$. In particular, $\sigma^{*}$ is stable on ideal factors of $S$. By Proposition 3.1, $\operatorname{Spec}^{\sigma^{*}}(S)$ is right link closed. Similarly, $\operatorname{Spec}^{\tau^{*}}(S)$ is left link closed. Thus, by Corollary 3.4, it suffices to show that $\operatorname{Spec}^{\sigma^{*}}(S)=\operatorname{Spec}^{\tau^{*}}(S)$.

Let $P \in \operatorname{Spec}^{\sigma^{*}}(S)$. Since $\sigma^{*}(S / P)=S / P, \sigma(S / P)=S / P$ and so $\sigma(R /(P \cap$ $R))=R /(P \cap R)$. Thus, if $Q_{1}, \ldots, Q_{n}$ denote all of the prime ideals of $R$ that are minimal over $P \cap R$, then each $Q_{i}$ is $\sigma$-dense. By hypothesis, each $Q_{i}$ is $\tau$ dense and hence $N=Q_{1} \cap \ldots \cap Q_{n}$ is also $\tau$-dense. Now, $N^{k} \subseteq P \cap R$ for some $k$. Consider the chain $R \supseteq N \supseteq \ldots \supseteq N^{k}$. Each factor $N^{i} / N^{i-1}$ and $R / N$ is $\tau$-torsion, whence $R / N^{k}$ is $\tau$-torsion. It follows that $R /(P \cap R)$ is $\tau$-torsion. By Lemma 4.2, $\tau^{*}(S / P)=S / P$. Therefore, $\operatorname{Spec}^{\sigma^{*}}(S) \subseteq \operatorname{Spec}^{\tau^{*}}(S)$. The reverse inclusion follows by the symmetric argument.

Using Corollary 3.4, we can view the last result as way of obtaining a link closed set of prime ideals in $S$ from a link closed set of prime ideals in $R$.

Corollary 4.4. Let $S \supseteq R$ be a Noetherian extension of a Noetherian ring $R$. Let $(\sigma, \tau)$ be a weakly symmetric pair for $R$. If $\operatorname{Sp}(\sigma, \tau)$ is link closed, then $\operatorname{Sp}\left(\sigma^{*}, \tau^{*}\right)$ is link closed.

\section{Invariance of classical Krull dimension}

Throughout, $\operatorname{Cldim}(R / I)$ will denote the classical Krull dimension of $R / I$ where $I \subseteq R$ is an ideal. For a Noetherian ring $R$, classical Krull dimension is called a link invariant provided $\operatorname{Cldim}(R / Q)=\operatorname{Cldim}(R / P)$ for $P, Q \in \operatorname{Spec}(R)$ with $Q \sim \rightarrow P$. The goal of this section is to show that, in this case, if $S$ is any Noetherian extension ring of $R$, then $\operatorname{Cldim}(R /(Q \cap R))=\operatorname{Cldim}(R /(P \cap R))$ for all prime ideals $P, Q \in \operatorname{Spec}(S)$ with $Q \sim \rightarrow P$.

From [5, Lemma 12.2], if $\beta \leq \operatorname{Cldim}(R)$, then there exists a prime ideal $P$ such that $\operatorname{Cldim}(R / P)=\beta$. Thus, for each ordinal $\beta \leq \operatorname{Cldim}(R)$, the set

$$
\operatorname{Spec}_{\beta}(R)=\{P \in \operatorname{Spec}(R) \mid \operatorname{Cldim}(R / P) \geq \beta\}
$$


is nonempty.

Define a weakly symmetric pair of torsion radicals $\left(\sigma_{\beta}, \tau_{\beta}\right)$ by declaring $\sigma_{\beta}$ to be the torsion radical cogenerated by

$$
\bigoplus\left\{{ }_{R} E(R / P) \mid P \in \operatorname{Spec}_{\beta}(R)\right\}
$$

and let $\tau_{\beta}$ to be its right-hand counterpart.

Clearly, if $P \in \operatorname{Spec}_{\beta}(R)$, then $\sigma_{\beta}(R / P)=\tau_{\beta}(R / P)=0$. Conversely, if $P$ is a prime ideal and $\sigma_{\beta}(R / P)=0$, then for some $P^{\prime} \in \operatorname{Spec}_{\beta}(R)$, there exists a nonzero homomorphism $f: R / P \rightarrow E\left(R / P^{\prime}\right)$. Let $H / P=f(R / P) \cap R / P^{\prime} \neq 0$. Then $P H \subseteq P^{\prime}$ forcing $P \subseteq P^{\prime}$. Thus, $\operatorname{Cldim}(R / P) \geq \operatorname{Cldim}\left(R / P^{\prime}\right) \geq \beta$ and so $P \in \operatorname{Spec}_{\beta}(R)$. A similar result holds on the right. Therefore for all $\beta \leq \operatorname{Cldim}(R)$,

$$
\operatorname{Spec}_{\sigma_{\beta}}(R)=\operatorname{Spec}_{\tau_{\beta}}(R)=\operatorname{Spec}_{\beta}(R) .
$$

Proposition 5.1. Let $R$ be a Noetherian ring for which classical Krull dimension is a link invariant. Then for all ordinals $\beta \leq \operatorname{Cldim}(R),\left(\sigma_{\beta}, \tau_{\beta}\right)$ is a weakly symmetric stable pair.

Proof. From the remark above, it's clear that $\operatorname{Sp}\left(\sigma_{\beta}, \tau_{\beta}\right)$ is link closed. The result follows from Corollary 3.4.

Theorem 5.2. Let $R$ be a Noetherian ring for which classical Krull dimension is a link invariant and let $S \supseteq R$ be a Noetherian extension ring. Then for all $P, Q \in \operatorname{Spec}(S)$ with $Q \sim \rightarrow P, \operatorname{Cldim}(R /(P \cap R))=\operatorname{Cldim}(R /(Q \cap R))$.

Proof. Pick $P, Q \in \operatorname{Spec}(S)$ with $Q \sim \rightarrow P$ and set $\beta=\operatorname{Cldim}(R /(P \cap R))$. Suppose that $\operatorname{Cldim}(R /(Q \cap R))<\beta$. For convenience, let $(\sigma, \tau)$ denote the pair $\left(\sigma_{\beta}, \tau_{\beta}\right)$.

If $I_{1}, \ldots, I_{n}$ are all of the distinct prime ideals of $R$ that are minimal over $Q \cap R$, then for all $j=1, \ldots, n, \operatorname{Cldim}\left(R / I_{j}\right)<\beta$ whence $I_{j} \notin \operatorname{Spec}_{\beta}(R)=\operatorname{Spec}_{\sigma}(R)$. Thus, for all $j, I_{j} \in \operatorname{Spec}^{\sigma}(R)$. By Lemma 4.2, $\sigma(R /(Q \cap R))=R /(Q \cap R)$.

Consider the pair $\left(\sigma^{*}, \tau^{*}\right)$. If $\sigma^{*}(S / P)=S / P$, then $\sigma(R /(P \cap R))=R /(P \cap$ $R)$. By [5, Lemma 12.2], there is a prime ideal $P^{\prime}$ of $R$ with $P^{\prime} \supseteq P \cap R$ and $\operatorname{Cldim}\left(R / P^{\prime}\right)=\operatorname{Cldim}(R /(P \cap R))=\beta$. However, since $\sigma\left(R / P^{\prime}\right)=R / P^{\prime}, P^{\prime} \notin$ $\operatorname{Spec}_{\beta}(R)=\operatorname{Spec}_{\sigma}(R)$ and hence $\operatorname{Cldim}\left(R / P^{\prime}\right)<\beta$, a contradiction. Therefore, $\sigma^{*}(S / P)=0$. It follows from Corollary 4.4 that $\sigma^{*}(S / Q)=0$. From the last paragraph, $\sigma(R /(Q \cap R))=R /(Q \cap R)$. Thus, by Lemma 4.2, $\sigma^{*}(S / Q)=S / Q$, another contradiction.

If $S \supseteq R$ is a ring extension, then a prime ideal $P$ of $S$ lies over a prime ideal $P^{\prime}$ of $R$ provided $P \cap R \subseteq P^{\prime}$ and $P^{\prime}$ is minimal over $P \cap R$. 
Corollary 5.3. Let $R$ be a Noetherian ring for which classical Krull dimension is a link invariant and let $S \supseteq R$ be a Noetherian extension ring that satisfies the following:

(1) If $P, Q \in \operatorname{Spec}(S)$ and $Q \subset P$, then $Q \cap R \subset P \cap R$.

(2) If $P, Q \in \operatorname{Spec}(S)$ and $Q \subset P$, then there is no $P^{\prime} \in \operatorname{Spec}(R)$ such that $P$ and $Q$ lie over $P^{\prime}$.

Then linked prime ideals in $S$ are incomparable.

Proof. Let $P, Q \in \operatorname{Spec}(S)$ with $Q \sim \rightarrow P$. If $Q \subset P$, then $Q \cap R \subset P \cap R$. By [5, Lemma 12.2], there is a prime ideal $P^{\prime}$ of $R$ with $P^{\prime} \supseteq P \cap R$ and $\operatorname{Cldim}\left(R / P^{\prime}\right)=$ $\operatorname{Cldim}(R /(P \cap R))$. It follows that $P^{\prime}$ is minimal over $P \cap R$. However, from Theorem 5.2, $\operatorname{Cldim}(R /(P \cap R))=\operatorname{Cldim}(R /(Q \cap R))$ and hence $P^{\prime}$ is also minimal over $Q \cap R$ contradicting part (2) of the hypothesis.

In the following, a ring $S$ is called a finite normalizing extension of a ring $R$ provided $S \supseteq R$ and there exists $a_{1}, \ldots, a_{n} \in S$ such that $R a_{i}=a_{i} R$ for all $i$ and $S=R a_{1}+\ldots+R a_{n}$. Strongly group graded rings are defined in [3] and extensively dealt with in [1].

Corollary 5.4. Let $R$ be a Noetherian ring for which classical Krull dimension is a link invariant. If $S$ is a finite normalizing extension of $R$ or if $S$ is a strongly $G$-graded extension of $R$ where $G$ is a finite group, then linked prime ideals in $S$ are incomparable.

Proof. In case $S$ is a finite normalizing extension of $R$, then according to $[9$, Proposition 2.12, Proposition 2.13], the first condition of Corollary 5.3 is true. From [8, Corollary 2.4], the second condition holds (in fact, for any finite extension).

For $S$ a strongly $G$-graded extension of $R$, it follows from [1, Proposition 3.7] that both (1) and (2) of Corollary 5.3 are true. The conclusion follows.

By [6, Theorem 8.2.8], classical Krull dimension is a link invariant for a Noetherian ring $R$ that satisfies the second layer condition. This is also true for any prime Noetherian ring $R$ with $\operatorname{Cldim}(R)=1$ since, in this case, no nonzero prime ideal can be linked to the zero ideal. However, not all such rings satisfy the second layer condition and, in general, classical Krull dimension is not a link invariant.

Example 5.5. Let $F$ be a field of characteristic 0, let $F(Y)$ be the field of rational functions and let $\delta$ be the usual derivative. Let $S=F(Y)[X ; \delta]$ and let $T$ be the subring $T=X S+F$. From [6, Example 1.3.20], $S$ is a simple principal ideal domain, $T$ is a Noetherian domain and $X S$ is the only nonzero proper ideal of $T$. 
It follows that $X S$ is idempotent. In fact, $X S$ is the only nonzero prime ideal of $T$ whence $\operatorname{Cldim}(T)=1$. Note that $\operatorname{Cldim}(S)=0$. From [9, 3.15], $T$ does not satisfy the second layer condition. Furthermore, by [6, Example 5.2.18], XS is torsion-free as a left $T$-module and as a right $S$-module. Consider the formal upper triangular matrix ring

$$
R=\left(\begin{array}{cc}
T & X S \\
0 & S
\end{array}\right)
$$

and the prime ideals $Q$ and $P$ defined below

$$
Q=\left(\begin{array}{cc}
0 & X S \\
0 & S
\end{array}\right), P=\left(\begin{array}{cc}
T & X S \\
0 & 0
\end{array}\right) .
$$

As rings, $R / Q \cong T$ while $R / P \cong S$. Also, $Q P=0$ and

$$
Q \cap P=\left(\begin{array}{cc}
0 & X S \\
0 & 0
\end{array}\right)
$$

Since $X S$ is torsion-free as a left $T$-module and as a right $S$-module, $Q \sim \rightarrow P$.

\section{References}

[1] A. D. Bell, Localization and ideal theory in Noetherian strongly group-graded rings, J. Algebra, 105(1) (1987), 76-115.

[2] J. L. Bueso, P. Jara and A. Verschoren, Compatability, Stability and Sheaves, Marcel Dekker, Inc., New York, 1995.

[3] E. C. Dade, Group-graded rings and modules, Math. Z., 174(3) (1980), 241-262.

[4] J. S. Golan, Localization of Noncommutative Rings, Marcel Dekker, Inc., New York, 1975.

[5] K. R. Goodearl and R. B. Warfield, An Introduction to Noncommutative Noetherian Rings, London Math. Soc. Student Texts, 16, Cambridge University Press, Cambridge, 1989.

[6] A. V. Jategaonkar, Localization in Noetherian Rings, London Math. Soc. Lecture Note Series, 98, Cambridge University Press, Cambridge, 1986.

[7] K. A. Kosler, On symmetric radicals over fully semiprimary Noetherian rings, J. Algebra Appl., 2(3) (2003), 351-364.

[8] E. S. Letzter, Prime ideals in finite extensions of Noetherian rings, J. Algebra, 135(2) (1990), 412-439.

[9] J. C. McConnell and J. C. Robson, Noncommutative Noetherian Rings, John Wiley and Sons, Ltd., Chichester, New York, 1987.

[10] B. Stenstrom, Rings of Quotients, Springer-Verlag, Berlin, New York, 1975. 
Karl A. Kosler

Department of Mathematics

University of Wisconsin-Waukesha

1500 University Drive

Waukesha, WI 53188, USA

e-mail: karl.kosler@uwc.edu 